\title{
Influence of Low-Degree High-Order $p$-Mode Splittings on the Solar Rotation Profile
}

\author{
R.A. García • S. Mathur · J. Ballot • A. Eff-Darwich • \\ S.J. Jiménez-Reyes · S.G. Korzennik
}

Received: 10 October 2007 / Accepted: 7 February 2008 / Published online: 4 March 2008

(C) Springer Science+Business Media B.V. 2008

\begin{abstract}
The solar rotation profile is well constrained down to about $0.25 R_{\odot}$ thanks to the study of acoustic modes. Since the radius of the inner turning point of a resonant acoustic mode is inversely proportional to the ratio of its frequency to its degree, only the low-degree $p$ modes reach the core. The higher the order of these modes, the deeper they penetrate into the Sun and thus they carry more diagnostic information on the inner regions. Unfortunately, the estimates of frequency splittings at high frequency from Sun-as-a-star measurements have higher observational errors because of mode blending, resulting in weaker
\end{abstract}

Helioseismology, Asteroseismology, and MHD Connections

Guest Editors: Laurent Gizon and Paul Cally.

R.A. García $(\bowtie) \cdot S$. Mathur

Laboratoire AIM, CEA/DSM-CNRS, U. Paris Diderot, IRFU/SAp, 91191 Gif-sur-Yvette Cedex, France

e-mail: rgarcia@cea.fr

S. Mathur

e-mail: smathur@cea.fr

J. Ballot

Max-Planck-Institut für Astrophysik, Karl-Schwarzschild-Strasse 1, 85748 Garching, Germany e-mail: jballot@mpa-garching.mpg.de

\section{A. Eff-Darwich}

Departamento de Edafología y Geología, Universidad de La Laguna, La Laguna, Tenerife, Spain e-mail: adarwich@ull.es

\section{A. Eff-Darwich · S.J. Jiménez-Reyes}

Instituto de Astrofísica de Canarias, 38205 La Laguna, Tenerife, Spain
A. Eff-Darwich
e-mail: adarwich@iac.es
S.J. Jiménez-Reyes
e-mail: sjimenez@iac.es

S.G. Korzennik

Harvard-Smithsonian Center for Astrophysics, 60 Garden Street, Cambridge, MA 02138, USA

e-mail: skorzennik@cfa.harvard.edu 
constraints on the rotation profile in the inner core. Therefore inversions for the solar internal rotation use only modes below $2.4 \mathrm{mHz}$ for $\ell \leq 3$. In the work presented here, we used an 11.5-year-long time series to compute the rotational frequency splittings for modes $\ell \leq 3$ using velocities measured with the GOLF instrument. We carried out a theoretical study of the influence of the low-degree modes in the region from 2 to $3.5 \mathrm{mHz}$ on the inferred rotation profile as a function of their error bars.

Keywords Helioseismology · Observations · Inverse modeling · Interior · Radiative zone · Core $\cdot$ Rotation

\section{Introduction}

Our knowledge of the solar rotation profile has been derived from the study of the resonant acoustic modes, which are trapped in the solar interior. Since the solar rotation lifts the azimuthal degeneracy of these resonant modes, their eigenfrequencies $\left(v_{n \ell m}\right)$ are split into their $m$ components, where $\ell$ is the angular degree, $n$ the radial order, and $m$ the azimuthal order. This separation $\Delta v_{n \ell m}$ - usually called rotational splitting (or just splitting) - depends on the rotation rate in the region sampled by the mode. Using inversion techniques, one can infer the rotation rate at different locations inside the Sun from a suitable linear combination of the measured rotational splittings

Today, the rotation rate inside the Sun is rather well known above $0.4 R_{\odot}$ (Thompson et al., 1996; Schou et al., 1998; Howe et al., 2000; Antia and Basu, 2000). The convective zone is characterized by a differential rotation extending from the surface down to the tachocline, located around $0.7 R_{\odot}$. Below the tachocline, inside the radiative region, the Sun appears to rotate as a rigid body with a nearly constant rate of $\approx 433 \mathrm{nHz}$ down to the solar core $\left(\right.$ i.e., $\left.\approx 0.25 R_{\odot}\right)$. The rotation rate inside the core derived from $p$ modes is still uncertain (Jiménez et al., 1994; Elsworth et al., 1995; Chaplin et al., 2001; Chaplin et al., 2004). Recent measurements of the asymptotic properties of the dipole $g$ modes and their comparison with solar models favors a faster rotation rate inside the solar core (García et al., 2007).

For $p$ modes of a given degree $\ell$, the radius at the inner turning point is a decreasing function of frequency, given by

$$
r_{\mathrm{t}}=c_{\mathrm{t}} L /\left(2 \pi v_{n \ell}\right),
$$

where $L=\ell+1 / 2, v_{n \ell}$ is the frequency of the mode, and $c_{\mathrm{t}}=c\left(r_{\mathrm{t}}\right)$ the sound-speed at the radius $r_{\mathrm{t}}$ (see, for example, Lopes and Turck-Chièze, 1994). Thus the modes with increasing frequencies - higher radial order $n$ - penetrate deeper inside the Sun. Unfortunately, when fitting Sun-as-a-star observations the uncertainties on the rotational splittings that are the most sensitive to low-degree $p$ modes are very large. Indeed, as the mode lifetimes decrease with frequency their line widths increase. Therefore, for frequencies above $\approx 2.3 \mathrm{mHz}$, there is a substantial blending among the visible $m$ components of the $p$ modes. This blending makes it difficult to extract precisely the rotational splitting. At higher frequencies $(\approx 3.9 \mathrm{mHz})$ even the successive pairs of $\ell=0,2$ and $\ell=1,3$ modes blend together. As a result, with today's fitting methods, it is still not possible to obtain values of the rotational splittings with an accuracy good enough to be useful in any rotation inversion.

By contrast, at low frequency - below $n=16$, or about $2.4 \mathrm{mHz}$ - the lifetime of the modes increases and thus their line width is very small. This allows us to measure their rotational splittings with very high precision. However, these modes have inner turning 
points at shallower depths than the high-frequency modes (above 0.08 and $0.12 R_{\odot}$ for the $\ell=1$ and 2 modes, respectively). Therefore, even though these modes do not carry any information below $\approx 0.1 R_{\odot}$, they help improve our knowledge of the inner rotation rate because their inclusion contributes to an increase of the precision of the inversions since they have smaller error bars (Eff-Darwich, Korzennik, and Jiménez-Reyes, 2002; Eff-Darwich et al., 2008).

For all of these reasons, rotation inversion methodologies usually limit the input data set of low-degree $p$ modes to low-frequency modes. For example, Couvidat et al. (2003) used a limited number of splittings, corresponding to modes with $\ell \leq 3$ and frequencies below $2.4 \mathrm{mHz}(n=15)$ resulting from fitting $\mathrm{GOLF}^{1}$ and $\mathrm{MDI}^{2} 2243$-day-long velocity time series, to infer the solar rotation profile. They concluded that the uncertainties in the rotation rate below $0.3 R_{\odot}$ were still quite large. Therefore, to obtain a better and more reliable rotation profile in the inner core, we need, on one hand, to include low-frequency acoustic, mixed, and gravity modes in the inversions (see discussions in Provost, Berthomieu, and Morel, 2000, and Mathur et al., 2008). On the other hand, we also need to measure more accurately low-degree high-order $p$ modes to further push the frequency limit of the modes used in the inversions.

We present, in Section 2, rotational splittings of $\ell \leq 3$ modes computed using 11.5 years of GOLF data, paying special attention that no bias is introduced by the solar activity or by the length of the fitting window. The length of these new time series improves the splitting error bars by $\sqrt{T}$ when compared to previous and shorter analyses with the same instrument. In Section 3, we study the sensitivity of the rotation rate below $0.25 R_{\odot}$ to the available $p$ modes and their error bars. We then discuss, in Section 4, the influence of these modes on the inversions, based on the resulting resolution kernels, and we finish by discussing the inverted rotation profile using the newly computed GOLF splittings.

\section{Data Analysis}

We analyzed a 4182-day-long time series of GOLF observations that spans the 11 April 1996 to 22 September 2007 epoch $(\approx 11.5$ years). These observations were calibrated by following the methods described in García et al. (2005). The duty cycle of that time series is $94.5 \%$. The GOLF instrument is a resonant-scattering spectrophotometer onboard the $\mathrm{SOHO}^{3}$ spacecraft. A standard fast Fourier transform algorithm was used to compute the power spectral density. This periodogram estimator was used because our primary interest is the study of modes in the medium-frequency range. We have therefore decided to privilege the simplest estimator, avoiding the use of multitapers or zero-padding estimators that are better suited to look for weak and narrow peaks at low frequency.

To obtain the mode parameters, each pair of modes $(\ell=0,2$ and $\ell=1,3)$ was fitted to a set of asymmetrical Lorentzians, as defined in Nigam and Kosovichev (1998), by using a maximum-likelihood method since the power-spectrum estimator follows a $\chi^{2}$ distribution with two degrees of freedom. For additional details on this method see, for example, Toutain and Appourchaux (1994). For each mode, we fitted the following set of parameters: the amplitude, the line width, the central frequency, the peak asymmetry, the rotational splitting (if $\ell>0$ ), and the background noise.

\footnotetext{
${ }^{1}$ Global Oscillations at Low Frequency (Gabriel et al., 1995).

${ }^{2}$ Michelson Doppler Imager (Scherrer et al., 1995).

${ }^{3}$ Solar and Heliospheric Observatory (Domingo, Fleck, and Poland, 1995).
} 


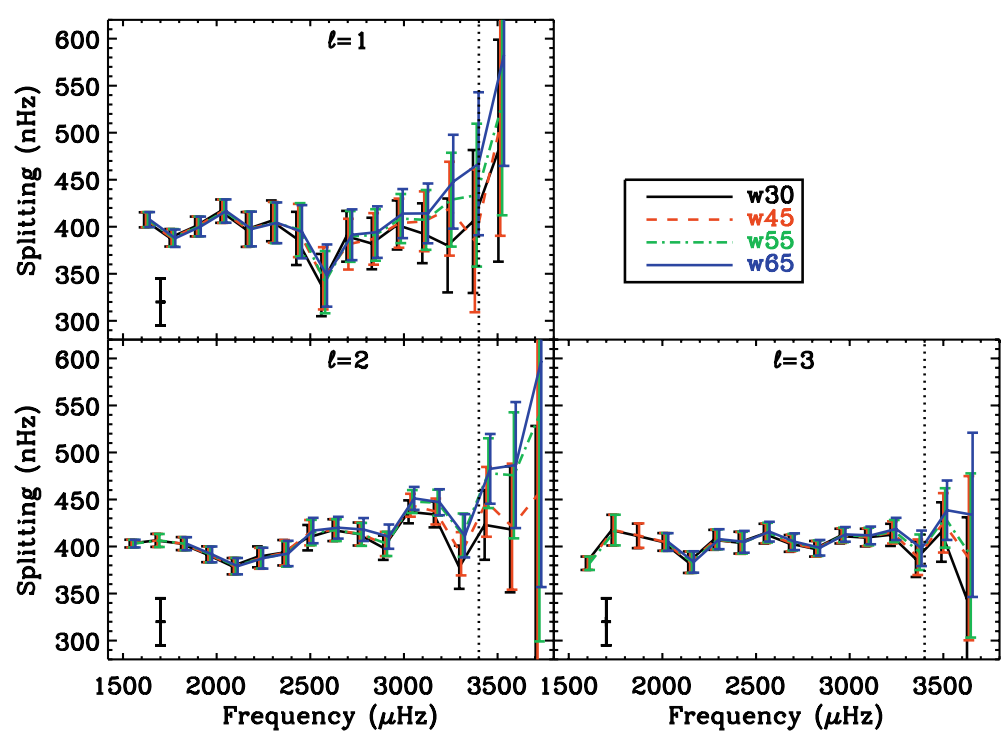

Figure 1 Synodic splittings for the $\ell=1,2$, and 3 modes fitted by using increasing fitting window widths from 30 to $65 \mu \mathrm{Hz}$. The vertical dotted lines mark the upper limit of the modes whose splittings are used in this work. The error bar in the bottom-left corner of each plot corresponds to $\pm 20 \mathrm{nHz}$ and is plotted as reference.

It has been shown that the amplitude ratios of the $m$ components inside a multiplet could produce a bias in the splitting determination if they are not correctly set (Chaplin et al., 2006). We have therefore fixed them to the averaged values obtained directly from the GOLF data set itself (for details see Henney, 1999). Finally, to reduce the parameter space, we used the same asymmetry and line width for both modes in any given fitting window. Indeed Chaplin et al. (2006) demonstrated that using the same width for the modes fitted simultaneously in a given fitting window reduces the bias in the splitting determination at high frequencies. Finally, the uncertainties on the fitted parameters were derived from the square root of the diagonal elements of the inverted Hessian matrix.

It has been shown, by using simulations, that the determination of the rotational splitting could be biased by the width of the fitting window (Chaplin et al., 2006). This is caused by the leakage of the neighboring modes. For example, the splittings of the $\ell=2$ modes could be noticeably modified (increased) at frequencies above $2.5 \mathrm{mHz}$ owing to the leakage of the $\ell=4$ modes. We have studied this effect using the GOLF data by selecting four different windows for the fit: 30, 45, 55, and $65 \mu \mathrm{Hz}$. The results are plotted in Figure 1. As expected, the values fitted for the $\ell=2$ splittings increase at high frequencies when wider fitting windows are used. This behavior is consistent with the results obtained by the solarFLAG group (see for comparison Figure 9 of Chaplin et al., 2006). However, the smallest window $(30 \mu \mathrm{Hz})$, also biases the fittings at high frequency because fitting such narrow spectral range prevents the fitting code from properly constraining the background level. Therefore, we have adopted the $45-\mu \mathrm{Hz}$ window as the best compromise between these two effects. In the case of the $\ell=1$ modes, the splittings computed using the smaller windows are roughly constant up to $3.4 \mathrm{mHz}$ and then increase. For higher frequencies the splittings increase whatever window width is used, and it is not possible to distinguish between a bias induced by the blending of the two adjacent $m$ components or a real increase. Therefore we have 

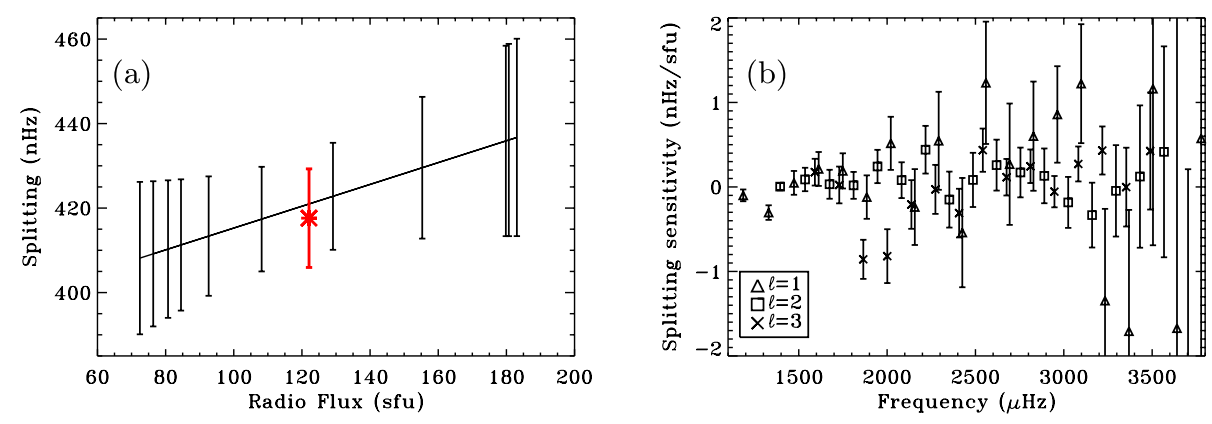

Figure 2 (a) Fitted splittings for the mode $(\ell=2, n=17)$ as a function of the solar activity level measured by the solar radio flux at $10.7 \mathrm{~cm}\left(1 \mathrm{sfu}=10^{-22} \mathrm{Wm}^{-2} \mathrm{~Hz}^{-1}\right)$. The asterisk with the thick error bar indicates the result of fitting the full time series. (b) Sensitivity of the splittings to the activity for all of the fitted modes $(\ell=1,2$, and 3$)$.

limited our study to the splittings of modes below $3.4 \mathrm{mHz}$ (a limit indicated with a vertical dotted line in Figure 1) where all of the splittings are roughly constant within the error bars. Another approach to limit the leakage of the neighbor peaks could be to fit the whole spectrum at once with a simple model of the background noise (Roca Cortés et al., 1998).

It is also important to notice that some splittings (for example, the $\ell=1, n=17$ mode) are slightly out of the general trend of the rest of the splittings (less than $2 \sigma$ ) as a consequence of the stochastic nature of the excitation. This deviation is in quantitative agreement with the results obtained from artificial simulations (see Chaplin et al., 2006).

We have also verified that there is no noticeable influence of solar activity on the extracted splittings. To do this, we followed an approach similar to the one described in JiménezReyes et al. (2001): We divided the time series into 12 independent 350-day-long segments ${ }^{4}$ and fitted them simultaneously. A linear dependence on a solar activity index was added to each parameter defining the spectral profiles, with two exceptions: First, we fixed the asymmetries to the values found by analyzing the full time series; second, we included a quadratic dependence on activity for the frequencies of the modes. We used the integrated $10.7-\mathrm{cm}$ radio flux measurements from the National Geophysical Data Center, ${ }^{5}$ averaged over the duration of each subseries as indicator of solar activity. The 10.7-cm radio flux has been shown to be the indicator that best correlates with the measured changes resulting from solar activity.

Figure 2(a) illustrates the variation of the splitting with solar activity for one mode $(\ell=2$, $n=17$ ), and Figure 2(b) shows the sensitivity of the splittings to the radio flux for all of the fitted modes (i.e., the slope in Figure 2(a)). This plot shows that there is no clear systematic dependence like the one seen for the frequency (Jiménez, Roca Cortés, and Jiménez-Reyes, 2002; Gelly et al., 2002; García et al., 2004b). Moreover variations during the solar cycle are generally marginally significant. The splittings obtained by fitting the whole time series are fully consistent with those obtained when using this activity-dependent method and correspond to the Sun at its mean activity level, as expected. The resulting rotational splittings for modes $\ell \leq 3$ are listed in Table 1 .

\footnotetext{
${ }^{4}$ One 350-day-long segment was not fitted since its duty cycle is very small because of the loss of contact with the SOHO spacecraft during that epoch.

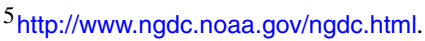


Table 1 Central frequencies $\left(v_{o}\right)$, synodic sectoral splittings $\left(\Delta v_{n \ell m}\right)$, and their respective $1 \sigma$ error bars $\left(\sigma_{o}\right.$ and $\left.\sigma_{n \ell m}\right)$ computed by using 4182 days of GOLF velocity time series.

\begin{tabular}{|c|c|c|c|c|c|}
\hline$\ell$ & $n$ & $v_{o}(\mu \mathrm{Hz})$ & $\sigma_{o}(\mathrm{nHz})$ & $\Delta v_{n \ell m}(\mathrm{nHz})$ & $\sigma_{n \ell m}(\mathrm{nHz})$ \\
\hline \multirow[t]{17}{*}{1} & 7 & 1185.5893 & 4.0 & 402.2 & 3.2 \\
\hline & 8 & 1329.6368 & 3.3 & 405.2 & 2.9 \\
\hline & 9 & 1472.8475 & 5.0 & 400.0 & 4.2 \\
\hline & 10 & 1612.7268 & 9.9 & 407.6 & 8.0 \\
\hline & 11 & 1749.2887 & 10.5 & 388.1 & 9.2 \\
\hline & 12 & 1885.0853 & 12.2 & 400.4 & 10.6 \\
\hline & 13 & 2020.8228 & 14.1 & 416.5 & 12.5 \\
\hline & 14 & 2156.8158 & 18.5 & 397.3 & 18.4 \\
\hline & 15 & 2292.0340 & 19.0 & 404.7 & 21.6 \\
\hline & 16 & 2425.6381 & 20.0 & 396.0 & 26.3 \\
\hline & 17 & 2559.2441 & 19.3 & 345.2 & 30.7 \\
\hline & 18 & 2693.4385 & 18.5 & 381.5 & 27.0 \\
\hline & 19 & 2828.2585 & 18.0 & 387.1 & 26.0 \\
\hline & 20 & 2963.4225 & 17.7 & 403.8 & 24.9 \\
\hline & 21 & 3098.2937 & 19.0 & 405.8 & 29.3 \\
\hline & 22 & 3233.2855 & 22.2 & 419.2 & 41.6 \\
\hline & 23 & 3368.6923 & 26.9 & 385.1 & 75.6 \\
\hline \multirow[t]{16}{*}{2} & 8 & 1394.6851 & 14.2 & 401.8 & 5.9 \\
\hline & 9 & 1535.8642 & 7.0 & 403.1 & 4.1 \\
\hline & 10 & 1674.5434 & 12.3 & 406.5 & 6.9 \\
\hline & 11 & 1810.3293 & 13.3 & 403.0 & 6.9 \\
\hline & 12 & 1945.8173 & 15.9 & 391.6 & 8.4 \\
\hline & 13 & 2082.1175 & 18.5 & 379.2 & 8.6 \\
\hline & 14 & 2217.6968 & 22.2 & 387.4 & 11.3 \\
\hline & 15 & 2352.2626 & 23.7 & 393.4 & 13.5 \\
\hline & 16 & 2485.9310 & 22.8 & 414.7 & 12.9 \\
\hline & 17 & 2619.7165 & 20.6 & 417.6 & 11.7 \\
\hline & 18 & 2754.5761 & 20.4 & 413.5 & 12.2 \\
\hline & 19 & 2889.6857 & 20.5 & 402.7 & 12.7 \\
\hline & 20 & 3024.8519 & 20.3 & 443.9 & 11.7 \\
\hline & 21 & 3159.9752 & 22.3 & 437.2 & 13.6 \\
\hline & 22 & 3295.2381 & 28.1 & 392.3 & 21.4 \\
\hline & 23 & 3430.9280 & 38.6 & 447.5 & 33.4 \\
\hline \multirow[t]{15}{*}{3} & 9 & 1591.4891 & 19.5 & 382.3 & 7.1 \\
\hline & 10 & 1729.1460 & 47.7 & 417.5 & 16.3 \\
\hline & 11 & 1865.3041 & 36.9 & 411.6 & 12.9 \\
\hline & 12 & 2001.2566 & 28.6 & 404.9 & 9.4 \\
\hline & 13 & 2137.7634 & 38.2 & 383.3 & 11.4 \\
\hline & 14 & 2273.4932 & 32.7 & 407.4 & 10.6 \\
\hline & 15 & 2407.6669 & 36.2 & 403.8 & 11.5 \\
\hline & 16 & 2541.7150 & 32.7 & 414.1 & 10.4 \\
\hline & 17 & 2676.2540 & 27.1 & 403.7 & 8.9 \\
\hline & 18 & 2811.4796 & 24.1 & 397.5 & 8.3 \\
\hline & 19 & 2947.1109 & 23.1 & 411.6 & 7.8 \\
\hline & 20 & 3082.4893 & 27.2 & 409.9 & 9.3 \\
\hline & 21 & 3217.9177 & 31.3 & 415.0 & 11.4 \\
\hline & 22 & 3353.6765 & 46.1 & 388.6 & 19.0 \\
\hline & 23 & 3489.6852 & 66.4 & 425.2 & 30.0 \\
\hline
\end{tabular}


Before inverting these rotational frequency splittings, we completed the set of splittings of low-degree low-order $p$ modes down to $1 \mathrm{mHz}$ with those extracted from the analysis of combined GOLF and MDI time series (García et al., 2004a). Then we added high-degree modes using splittings of modes between $\ell=4$ and $\ell=25$ from the analysis of the 2088day-long time series of MDI observations fitted by Korzennik (2005).

\section{Sensitivity of the Splittings to the Rotation Rate below $0.25 R_{\odot}$}

We have shown in the introduction that the acoustic modes of higher radial order penetrate deeper in the solar interior and that they are potentially of great interest to better constrain the rotation inside the solar core. This is illustrated in Figure 3, where the modes listed in Table 1 are plotted as a function of the radius of their inner turning point.

The vertical error bars correspond to the $1 \sigma$ error bars of the splittings. These uncertainties are smaller for the $\ell=2$ and 3 than for the $\ell=1$ modes because the visible $m$ components of the latter are closer in frequency and therefore blend together at lower frequencies than for higher degree modes.

The amount of information on the rotation rate inside the core $\left(r \leq 0.25 R_{\odot}\right)$ present in these modes depends on the precision of the measured splittings. The sensitivity of the splittings to the rotation rate below $0.25 R_{\odot}$ is plotted in Figure $4(\mathrm{a})$. This sensitivity is defined as the ratio of the contribution of the rotation rate below $0.25 R_{\odot}$ to the splitting $\left(\Delta v_{n \ell m}\right)$ and the uncertainty $\left(\sigma_{n \ell m}\right)$ in its determination [see Equation (1) for sectoral modes, $\ell=m]$ :

$$
\text { Sensitivity }=\left(\frac{\int_{0}^{0.25 R_{\odot}} K_{n, \ell}(r) \Omega(r) \mathrm{d} r}{\int_{0}^{R_{\odot}} K_{n, \ell}(r) \Omega(r) \mathrm{d} r} \Delta v_{n, \ell}\right) / \sigma_{n, \ell} .
$$

For a given mode, if the sensitivity function is greater than one, this particular mode provides useful information on the core rotation. Up to $\approx 3.4 \mathrm{mHz}$, all of the modes $\ell \leq 3$ are potentially interesting for the inversions. The situation has evolved drastically since 2003 when the previous analysis of 2243-day-long GOLF and MDI time series showed that below $0.2 R_{\odot}$ only those modes below $2.2 \mathrm{mHz}$ had a sensitivity greater than one (see the left panel of Figure 1 in Couvidat et al., 2003). In fact, when we compute the sensitivity function below the latter radius using the new computed GOLF splittings we obtain the same dependence

Figure 3 Fitted modes listed in Table 1 as a function of the inner turning point radii. The error bars are the splitting error bars in nanohertz magnified by a factor of $10^{4}$.

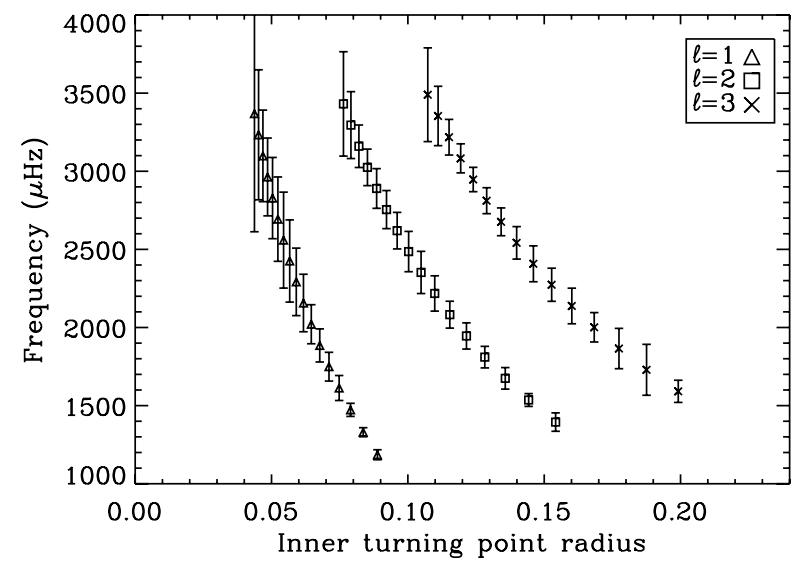



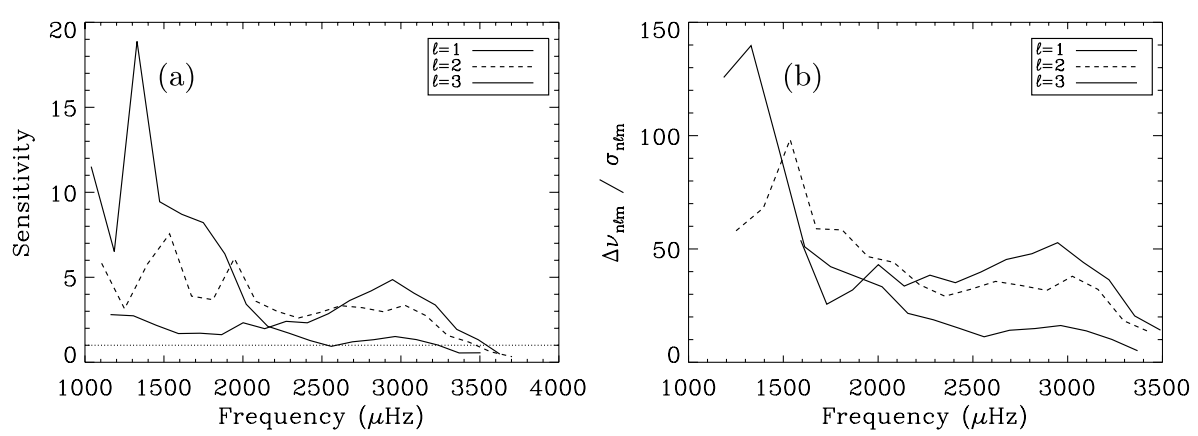

Figure 4 (a) Sensitivity of the splittings to the rotation rate below $0.25 R_{\odot}$ as defined in the text. The horizontal dotted line is the lower limit below which the splittings would not carry any useful information on the solar core rotation. (b) Splittings normalized by their error bars.

with the frequency below $0.25 R_{\odot}$ but rescaled to lower sensitivity. For the $\ell=2$ and 3 modes the sensitivity reduction is about a factor of two. In the case of the $\ell=1$ modes, the factor is smaller and the sensitivity curve crosses unity at 3.1 instead of $3.3 \mathrm{mHz}$.

Figure 4(b) shows the resulting splittings normalized by their error bars. The precision clearly increases toward the low-frequency range for the $\ell=1$ modes whereas for the $\ell=2$ and 3 modes the curves are flatter, indicating that the error bars are nearly constant all along the analyzed frequency range.

\section{Discussion}

To study the effect of the $\ell \leq 3$ modes at medium-range frequencies on the inferred rotation rate we carry out some $2 \mathrm{D}$ inversions.

The rotational splittings $\left(\Delta v_{n \ell m}\right)$ are the integral of the product of a sensitivity function or a kernel $-K_{n \ell m}(r, \theta)$, a known function based on a solar model, with the rotation rate $[\Omega(r, \theta)]$ over the radius $(r)$ and the co-latitude $(\theta)$ (Hansen, Cox, and van Horn, 1977):

$$
\Delta v_{n \ell m}=\frac{1}{2 \pi} \int_{0}^{R} \int_{0}^{\pi} K_{n \ell m}(r, \theta) \Omega(r, \theta) \mathrm{d} r \mathrm{~d} \theta+\epsilon_{n \ell m},
$$

where $\epsilon_{n \ell m}$ is the effective error on the measured value $\Delta v_{n \ell m}$. We assume that the errors $\left(\epsilon_{n \ell m}\right)$ follow a normal distribution with a standard deviation $\sigma_{n \ell m}$, which is estimated by the fitting procedure (see Section 2), as listed in Table 1. This set of equations defines a classical inverse problem for the solar rotation. The inversion of this set of $M$ integral equations one for each measured $\Delta v_{n \ell m}$ - allows us to infer the rotation rate profile as a function of radius and latitude from a set of observed splittings.

The inversion method we use here is based on the regularized least-squares methodology (RLS) following the prescription described in Eff-Darwich and Pérez Hernández (1997). In this implementation, the regularization function is weighted differently for each model grid point (see also Eff-Darwich et al., 2008). In summary, Equation (2) is transformed into a matrix relation,

$$
\mathbf{D}=\boldsymbol{A} \mathbf{x}+\epsilon,
$$


where $\mathbf{D}$ is the data vector, with elements $\Delta v_{n \ell m}$ and dimension $M, \mathbf{x}$ is the solution vector to be determined at $N$ model grid points, $\boldsymbol{A}$ is the matrix with the kernels, of dimension $M \times N$, and $\epsilon$ is the vector containing the error bars.

The RLS solution for the vector $\mathbf{x}$ is given by

$$
\mathbf{x}_{\mathrm{est}}=\left(\boldsymbol{A}^{\mathrm{T}} \boldsymbol{A}+\gamma \boldsymbol{H}\right)^{-1} \boldsymbol{A}^{\mathrm{T}} \mathbf{D},
$$

where $\gamma$ is a scalar introduced to give a suitable weight to the constraint matrix $\boldsymbol{H}$ on the solution. Replacing D from Equation (3) we obtain

$$
\mathbf{x}_{\mathrm{est}}=\left(\boldsymbol{A}^{\mathrm{T}} \boldsymbol{A}+\gamma \boldsymbol{H}\right)^{-1} \boldsymbol{A}^{\mathrm{T}} \boldsymbol{A} \mathbf{x} \equiv \boldsymbol{R} \mathbf{x}
$$

and hence

$$
\boldsymbol{R}=\left(\boldsymbol{A}^{\mathrm{T}} \boldsymbol{A}+\gamma \boldsymbol{H}\right)^{-1} \boldsymbol{A}^{\mathrm{T}} \boldsymbol{A} .
$$

The matrix $\boldsymbol{R}$ is referred to as the resolution or sensitivity matrix. Ideally, $\boldsymbol{R}$ would be the identity matrix, which corresponds to perfect resolution. However, if we try to find an inverse with a resolution matrix $\boldsymbol{R}$ close to the identity matrix, the solution is generally dominated by noise magnification. The individual columns of $\boldsymbol{R}$ display how anomalies in the corresponding model are imaged by the combined effect of measurement and inversion. In this sense, each element $R_{i j}$ reveals how much of the anomaly in the $j$ th inversion model grid point is transferred into the $i$ th grid point. Consequently, the diagonal elements $R_{i i}$ indicate how much of the information is saved in the model estimate and may be interpreted as the resolvability or sensitivity of $x_{i}$. We defined the sensitivity $\lambda_{i}$ of the grid point $x_{i}$ to the inversion process as follows:

$$
\lambda_{i}=\frac{R_{i i}}{\sum_{j=1}^{N} R_{i j}} .
$$

With this definition, a lower value of $\lambda_{i}$ means a lower sensitivity of $x_{i}$ to the inversion of the solar rotation. We define a smoothing vector $\mathbf{W}$ with elements $w_{i}=\lambda_{i}^{-1}$ that is introduced in Equation (4) to complement the smoothing parameter $\gamma$, namely

$$
\mathbf{x}_{\text {est }}=\left(\boldsymbol{A}^{\mathrm{T}} \boldsymbol{A}+\gamma \mathbf{W} \boldsymbol{H}\right)^{-1} \boldsymbol{A}^{\mathrm{T}} \mathbf{D} .
$$

Such substitution allows us to apply different regularizations to different model grid points $x_{i}$, whose sensitivities depend on the data set that is used in the inversions. A set of results can be calculated for different values of $\gamma$, the optimal solution being the one with the best trade-off between error propagation and the quadratic difference $\chi^{2}=\left|\boldsymbol{A} \mathbf{x}_{\text {est }}-\mathbf{D}\right|^{2}$ as discussed in Eff-Darwich and Pérez Hernández (1997).

We have performed a theoretical study to determine the effect of adding the low-degree high-order $p$ modes in the inversions. To do so, we have computed, using Equation (2), the splittings corresponding to an artificial rotation profile $[\Omega(r, \theta)]$. This artificial profile has a differential rotation in the convection zone (that mimics the real one), a rigid rotation from 0.7 down to $0.2 R_{\odot}$ equal to $\Omega_{\mathrm{rz}}=433 \mathrm{nHz}$, and a steplike profile in the core having a rate of $350 \mathrm{nHz}$ in the $0.1-0.2 R_{\odot}$ region and a rate three times larger than the rest of the radiative zone below $0.1 R_{\odot}$. Although this profile with steep changes and a small drop followed by an increase is unlikely to be realistic, it enables us to characterize the quality of the inversions as the inversion code has difficulty reproducing these steep gradients. 
Table 2 Description of the artificial data sets used to study the sensitivity of low-degree high-order $p$ modes.

\begin{tabular}{llll}
\hline Data set & $\ell=1(\mathrm{mHz})$ & $\ell=2,3(\mathrm{mHz})$ & $\ell>3(\mathrm{mHz})$ \\
\hline 1 & $1 \leq v \leq 2.3$ & $1 \leq v \leq 2.3$ & $1 \leq v \leq 3.9$ \\
2 & $1 \leq v \leq 2.5$ & $1 \leq v \leq 3.4$ & $1 \leq v \leq 3.9$ \\
3 & $1 \leq v \leq 3.4$ & $1 \leq v \leq 3.4$ & $1 \leq v \leq 3.9$ \\
\hline
\end{tabular}

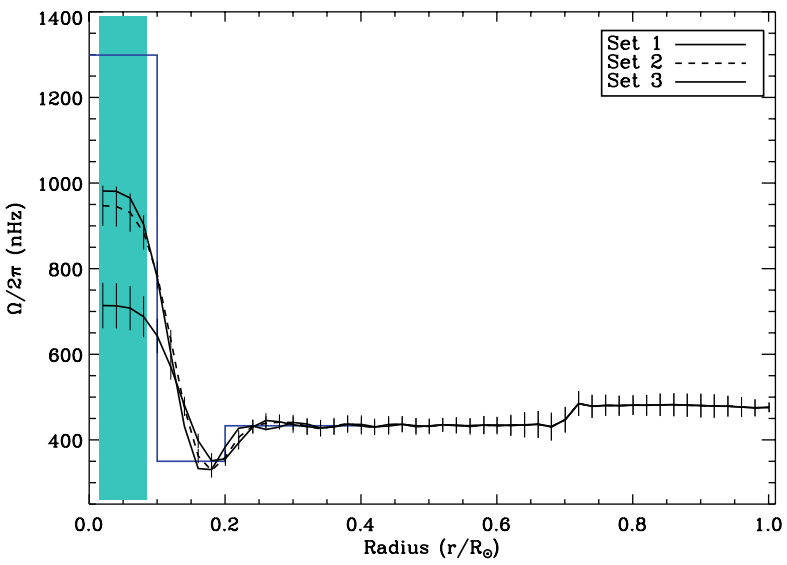

Figure 5 Equatorial rotation rate obtained from ideal inversions (all the modes with the same error bars) of an artificial step profile (continuous line) by using the three data sets described in Table 2. The inversion error bars are plotted only for the profiles resulting from inverting Sets 1 and 2. The shaded area corresponds to the region where the solution of the inversion is not reliable because the lack of sensitivity of the acoustic modes to this region of the Sun gives poor-quality resolution kernels.

Three artificial data sets have been built (see Table 2). The first, Set 1, is the reference and contains low-degree $p$ modes below $2.3 \mathrm{mHz}$ whereas $p$ modes for $\ell>3$ extend up to $3.9 \mathrm{mHz}$. The second set, Set 2 , adds the $\ell=2$ and 3 modes up to $3.4 \mathrm{mHz}$. As we have shown in the previous section, these modes could have some sensitivity to the rotation in the core (see Figure 4(a)). Finally, the third set, Set 3, also adds the $\ell=1$ modes up to $3.4 \mathrm{mHz}$ that seem to be at the sensitivity limit (see again Figure 4(a)).

Figure 5 shows the resulting inversions for these three artificial data sets. We shall qualify these inversions as ideal because all of the splittings were given the same error bars and are noiseless. The inferred rotation rate represents the best result that we can obtain for each set of modes.

As expected, the differences between the profiles appear below $0.3 R_{\odot}$ but they are only significant below $\approx 0.15 R_{\odot}$. All through the solar interior, the differences between Sets 2 and 3 are very small and are within the inversion uncertainties. Comparing these two data sets with Set 1 , we obtain an improvement up to $\approx 30 \%$ in the deepest region when the sets with more modes are used. Unfortunately, in all of the data sets, the recovered profiles are not accurate enough. This is easily understood by looking at the corresponding resolution kernels, which remain, indeed, very broad. Figure 6 shows some resolution kernels for the deepest model grid points and for Sets 1 and 3. There are no resolution kernels centered below $\approx 0.13 R_{\odot}$ for Set 1 . Comparing the resolution kernels of Sets 1 and 3, we see an improvement in the second case. Indeed the resolution kernels are narrower (better resolution in the solution of the inversion) and they are slightly shifted to deeper layers (by about 3\% of the solar radius).

We have shown the improvements of adding low-degree high-order $p$ modes in the ideal inversions. However, the observed splittings have nonuniform error bars. Therefore, we inverted the same sets of artificial splittings but using the actual, measured error bars. To be more realistic, Gaussian noise was also added to the artificial splittings, commensurate with their corresponding uncertainty. The resulting rotation profiles are plotted in Figure 7. As 
Figure 6 Resolution kernels of the inner model grid for Set 1 (top) and 3 (bottom) in the ideal case (where all the modes have the same error bars).

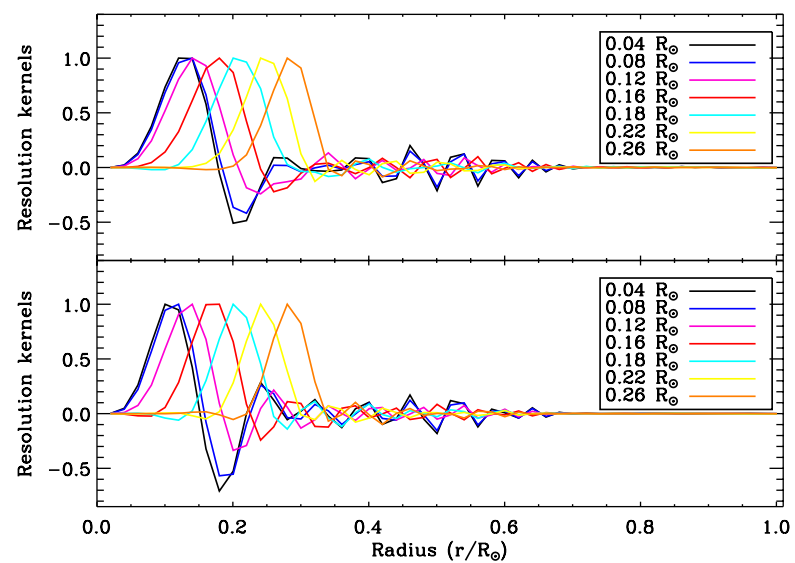

Figure 7 Equatorial rotation rate obtained from the inversions of the artificial step profile (continuous line) by using the three data sets described in Table 2 but with the real error bars as described in Section 2. For clarity, the inversion error bars are plotted only on the inversion of the Set 2 . The shaded area corresponds to the region where the solution of the inversion is not reliable, as explained in Figure 5.

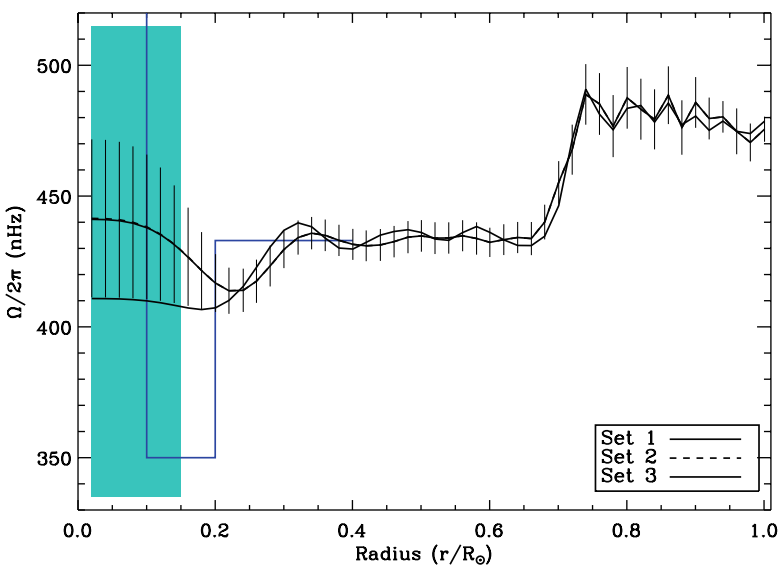

for the ideal case, Sets 2 and 3 give the same qualitative results and both are slightly better in the core than Set 1, even though the differences are within the inversion error bars. Nevertheless, the solution is improved in all of the radiative region where the ripples are reduced and the solution is smoother when more modes are added. Therefore, even with the realistic uncertainties, the recovered rotation rate is more accurate when Sets 2 or 3 are used. Unfortunately, the contribution of the $\ell=1$ modes in the $2.5-3.4 \mathrm{mHz}$ range remains negligible with the present magnitude of their uncertainties.

Including realistic uncertainties results in significant changes in the resolution kernels (see Figure 8). Indeed, the coefficients of the linear combination given to each splitting (inversely proportional to the error bars) changes the linear combination of the modes used to compute those resolution kernels. Therefore the inner resolution kernels shift outward: The maxima of the deepest one are now at around $0.16 R_{\odot}$ for all sets and, of course, are broader than the ones of the ideal case. The differences between the resolution kernels deduced from Sets 1 and 3 are smaller, explaining why the solutions in both cases are so similar.

After having studied the improvements in the rotation rate that we can expect by adding the low-degree high-order $p$ modes, we computed the same set of inversions using the real data. Figure 9 shows the inferred rotation rate for the real data using the Sets 1, 2, and 3. The results are similar to what we found for the artificial cases with realistic uncertainties. 
Figure 8 Resolution kernels of the inner model grid points for Set 1 (top) and 3 (bottom) but by using splittings with realistic uncertainties.
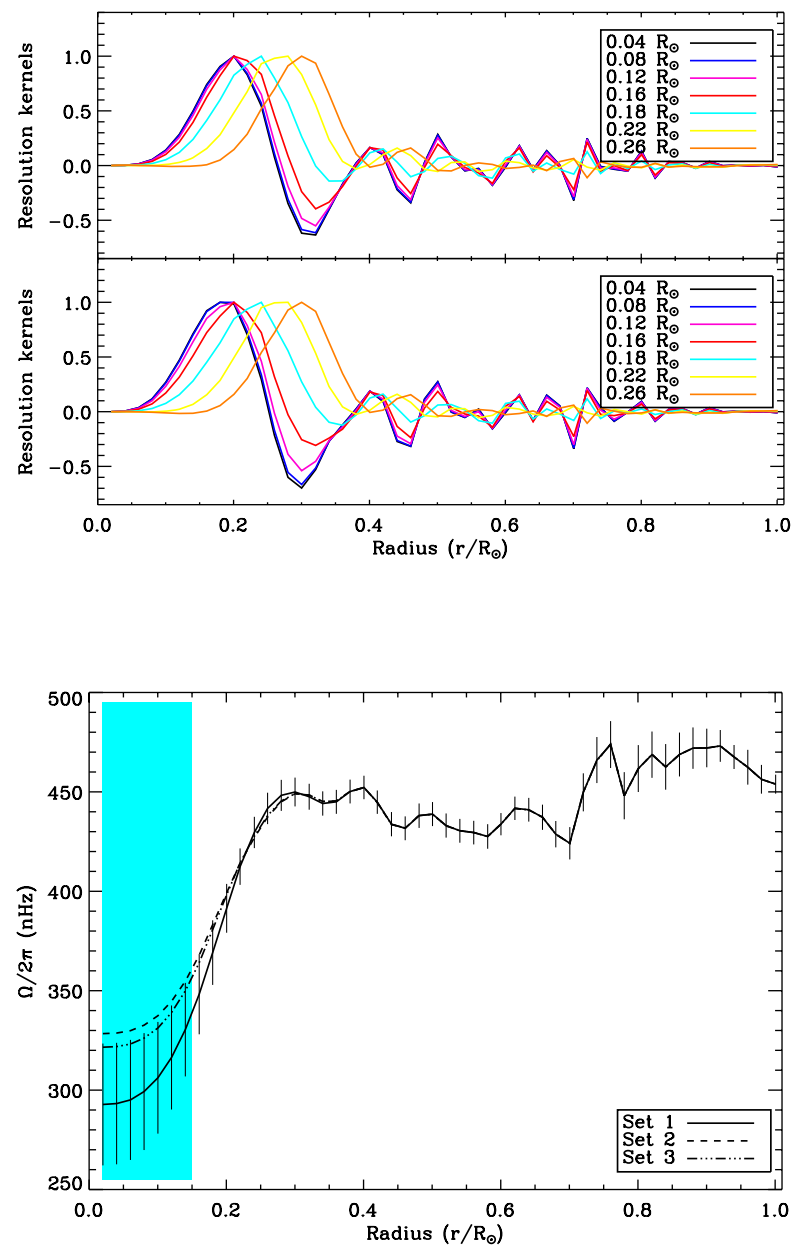

Figure 9 Equatorial rotation rate of real data by using only low-degree low-order $p$ modes up to 2.3 and $3.4 \mathrm{mHz}$ and the same set of high-degree modes. For clarity, the inversion error bars are shown only for the result of inverting Set 1 . The shaded area corresponds to the region where the solution of the inversion is not reliable as explained in Figure 5.

Indeed, the rotation rate below $\approx 0.25 R_{\odot}$ changes slightly when one compares Sets 2 and 3 (which cannot be distinguished within the error bars) to Set 1 . The rotation profiles show a small increase inside the core when the new modes are added (Sets 2 and 3 ). The difference is barely significant down to $0.16 R_{\odot}$, which is the reliability limit of the inversion. As we already knew, we need gravity modes to get more information about the dynamics of the deepest layers of the core (Mathur et al., 2008). In the rest of the radiative zone (above $\left.0.25 R_{\odot}\right)$, the profiles inferred by using the three sets are the same.

We have also tested the effect of increasing the amplitude ratio of the visible $m$ components of the $\ell=2$ and 3 modes by $20 \%$ and $10 \%$ respectively. The resulting splittings are systematically increased by $\approx 10 \mathrm{nHz}$ for the $\ell=2$ modes and by 3 to $4 \mathrm{nHz}$ for the $\ell=3$ modes. The inversion computed with these new splittings gives the same qualitative results as those shown in Figure 9: The inferred profiles are improved when more modes are used (Sets 2 and 3). For these two profiles, the rotation rate obtained at $0.16 R_{\odot}$ has increased by some $7 \%$, which is still within the inversion error bars. 


\section{Conclusion}

We have analyzed a 4182-day-long time series of GOLF velocity observation and have derived the frequencies and the splittings of the acoustic modes up to $3.4 \mathrm{mHz}$. We used a $45-\mu \mathrm{Hz}$ window to fit the $\ell=0,2$ and 1,3 pairs to limit any bias in the fitted splittings. By fitting shorter time series simultaneously and including a linear dependence of the splitting with solar activity, we have checked that for most of the modes the variation of this parameter with the activity is within the error bars estimated by using the whole time series.

We then carried out a study of the sensitivity of the rotation of the core (below $0.25 R_{\odot}$ ) to the $\ell \leq 3$ modes up to $3.4 \mathrm{mHz}$. We show that the contribution of the rotation rate in the core to the splittings of the $\ell=2$ and 3 modes is several times the size of their current error bars. By contrast the $\ell=1$ modes in the $2.5-3.4 \mathrm{mHz}$ range have a contribution from the core of the same order of magnitude as their error bars. Therefore, they cannot provide valuable information on the core's rotation.

By using artificial inversions of a steplike profile we have shown that, ideally, the new modes would improve the inferred rotation rate in the core but with a small accuracy on the profile itself owing to a lack of resolution in the inner regions of the Sun - as indicated by the corresponding resolution kernels. The effect of including the $\ell=1$ modes between 2.5 and $3.4 \mathrm{mHz}$ is negligible with the current size of their error bars. Using the real error bars degrades the inferred solution and the differences in the core profile are within the inversion uncertainties. However, the profile in the rest of the radiative region is smoother when more modes are used, demonstrating that the inclusion of those modes improves the inversion results.

Finally, the inversion of the real data, including the larger set of low-degree low-order $p$ modes, shows no significant differences between Sets 2 and 3 as for the artificial data. There is a small increase of the rotation rate below $0.25 R_{\odot}$ compared to the reference Set 1 but it is within the inversion error bars. The resolution kernels are improved when the lowdegree high-order $p$ modes are included and the resolution kernels at the deepest locations are shifted by $3 \%$ of the solar radius toward the center. This result suggests that with the present error bars it is better to use such modes in the inversions (at least for the high-order $\ell=2$ and 3 modes).

To make progress in the inference of the rotation rate in the solar core we would need to measure the low-degree modes - acoustic, mixed, and gravity modes - that will bring a direct signature of the dynamics of the still hidden core of the Sun.

Acknowledgements The GOLF experiment is based upon a consortium of institutes (IAS, CEA/Saclay, Nice, and Bordeaux Observatories from France and IAC from Spain) involving numerous scientists and engineers, as enumerated in Gabriel et al. (1995). SOHO is a mission of international cooperation between ESA and NASA. This work has been partially funded by Grant No. AYA2004-04462 of the Spanish Ministry of Education and Culture and partially supported by the European Helio- and Asteroseismology Network $\left(\right.$ HELAS $^{6}$ ), a major international collaboration funded by the European Commission's Sixth Framework Programme.

\section{References}

Antia, H.M., Basu, S.: 2000, Temporal variations of the rotation rate in the solar interior. Astrophys. J. 541, 442 - 448. doi:10.1086/309421.

\footnotetext{
${ }^{6}$ http://www.helas-eu.org/.
} 
Chaplin, W.J., Elsworth, Y., Isaak, G.R., Marchenkov, K.I., Miller, B.A., New, R.: 2001, Rigid rotation of the solar core? On the reliable extraction of low- $l$ rotational $p$-mode splittings from full-disc observations of the sun. Mon. Not. Roy. Astron. Soc. 327, 1127 - 1136. doi:10.1046/j.1365-8711.2001.04805.x.

Chaplin, W.J., Sekii, T., Elsworth, Y., Gough, D.O.: 2004, On the detectability of a rotation-rate gradient in the solar core. Mon. Not. Roy. Astron. Soc. 355, 535 - 542. doi:10.1111/j.1365-2966.2004.08338.x.

Chaplin, W.J., Appourchaux, T., Baudin, F., Boumier, P., Elsworth, Y., Fletcher, S.T., Fossat, E., García, R.A., Isaak, G.R., Jiménez, A., Jiménez-Reyes, S.J., Lazrek, M., Leibacher, J.W., Lochard, J., New, R., Pallé, P., Régulo, C., Salabert, D., Seghouani, N., Toutain, T., Wachter, R.: 2006, Solar FLAG hare and hounds: on the extraction of rotational $p$-mode splittings from seismic, sun-as-a-star data. Mon. Not. Roy. Astron. Soc. 369, 985 -996. doi:10.1111/j.1365-2966.2006.10358.x.

Couvidat, S., García, R.A., Turck-Chièze, S., Corbard, T., Henney, C.J., Jiménez-Reyes, S.: 2003, The rotation of the deep solar layers. Astrophys. J. 597, L77 - L79. doi:10.1086/379698.

Domingo, V., Fleck, B., Poland, A.I.: 1995, The SOHO mission: an overview. Solar Phys. 162, 1 - 37.

Eff-Darwich, A., Pérez Hernández, F.: 1997, A new strategy for helioseismic inversions. Astron. Astrophys. Suppl. 125, $391-398$.

Eff-Darwich, A., Korzennik, S.G., Jiménez-Reyes, S.J.: 2002, Inversion of the internal solar rotation rate. Astrophys. J. 573, 857-863. doi:10.1086/340747.

Eff-Darwich, A., Korzennik, S.G., Jiménez-Reyes, S.J., García, R.A.: 2008, The rotation of the solar radiative interior after 2088 days of helioseismic observations from GONG, GOLF and MDI. Astrophys. J. in press. http://arXiv.org/abs/0802.3604.

Elsworth, Y., Howe, R., Isaak, G.R., McLeod, C.P., Miller, B.A., New, R., Wheeler, S.J., Gough, D.O.: 1995, Slow rotation of the sun's interior. Nature 376, 669-672. doi:10.1038/376669a0.

Gabriel, A.H., Grec, G., Charra, J., Robillot, J.M., Cortés, T.R., Turck-Chièze, S., Bocchia, R., Boumier, P., Cantin, M., Céspedes, E., Cougrand, B., Cretolle, J., Dame, L., Decaudin, M., Delache, P., Denis, N., Duc, R., Dzitko, H., Fossat, E., Fourmond, J.J., García, R.A., Gough, D., Grivel, C., Herreros, J.M., Lagardere, H., Moalic, J.P., Pallé, P.L., Petrou, N., Sanchez, M., Ulrich, R., van der Raay, H.B.: 1995, Global oscillations at low frequency from the SOHO mission (GOLF). Solar Phys. 162, 61 - 99.

García, R.A., Corbard, T., Chaplin, W.J., Couvidat, S., Eff-Darwich, A., Jiménez-Reyes, S.J., Korzennik, S.G., Ballot, J., Boumier, P., Fossat, E., Henney, C.J., Howe, R., Lazrek, M., Lochard, J., Pallé, P.L., Turck-Chièze, S.: 2004a, About the rotation of the solar radiative interior. Solar Phys. 220, 269-285. doi:10.1023/B:SOLA.0000031395.90891.ce.

García, R.A., Jiménez-Reyes, S.J., Turck-Chièze, S., Ballot, J., Henney, C.J.: 2004b, Solar low-degree $p$ mode parameters after 8 years of velocity measurements with SOHO. In: Danesy, D. (ed.) ESA SP-559: SOHO 14 Helio- and Asteroseismology: Towards a Golden Future 14, ESA, Noordwijk, 436.

García, R.A., Turck-Chièze, S., Boumier, P., Robillot, J.M., Bertello, L., Charra, J., Dzitko, H., Gabriel, A.H., Jiménez-Reyes, S.J., Pallé, P.L., Renaud, C., Roca Cortés, T., Ulrich, R.K.: 2005, Global solar Doppler velocity determination with the GOLF/SoHO instrument. Astron. Astrophys. 442, $385-395$. doi:10.1051/0004-6361:20052779.

García, R.A., Turck-Chièze, S., Jiménez-Reyes, S.J., Ballot, J., Pallé, P.L., Eff-Darwich, A., Mathur, S., Provost, J.: 2007, Tracking solar gravity modes: The dynamics of the solar core. Science 316, $1591-$ 1593. doi:10.1126/science.1140598.

Gelly, B., Lazrek, M., Grec, G., Ayad, A., Schmider, F.X., Renaud, C., Salabert, D., Fossat, E.: 2002, Solar $p$-modes from 1979 days of the GOLF experiment. Astron. Astrophys. 394, $285-297$. doi:10.1051/0004-6361:20021106.

Hansen, C.J., Cox, J.P., van Horn, H.M.: 1977, The effects of differential rotation on the splitting of nonradial modes of stellar oscillation. Astrophys. J. 217, 151-159.

Henney, C.J.: 1999, Comparison between simultaneous golf and mdi observations in search of low frequency solar oscillations. Ph.D. thesis, University of California, Los Angeles.

Howe, R., Christensen-Dalsgaard, J., Hill, F., Komm, R.W., Larsen, R.M., Schou, J., Thompson, M.J., Toomre, J.: 2000, Dynamic variations at the base of the solar convection zone. Science 287, 24562460.

Jiménez, A., Roca Cortés, T., Jiménez-Reyes, S.J.: 2002, Variation of the low-degree solar acoustic mode parameters over the solar cycle. Solar Phys. 209, 247-263.

Jiménez, A., Pérez Hernandez, F., Claret, A., Pallé, P.L., Régulo, C., Roca Cortés, T.: 1994, The rotation of the solar core. Astrophys. J. 435, 874-880. doi:10.1086/174868.

Jiménez-Reyes, S.J., Corbard, T., Pallé, P.L., Roca Cortés, T., Tomczyk, S.: 2001, Analysis of the solar cycle and core rotation using 15 years of Mark-I observations: 1984-1999. I. The solar cycle. Astron. Astrophys. 379, 622 - 633. doi:10.1051/0004-6361:20011374.

Korzennik, S.G.: 2005, A mode-fitting methodology optimized for very long helioseismic time series. Astrophys. J. 626, 585 -615. doi:10.1086/429748. 
Lopes, I., Turck-Chièze, S.: 1994, The second order asymptotic theory for the solar and stellar low degree acoustic mode predictions. Astron. Astrophys. 290, $845-860$.

Mathur, S., Eff-Darwich, A.M., García, R.A., Turck-Chièze, S.: 2008, Sensitivity of helioseismic gravity modes to the dynamics of the solar core. Astron. Astrophys. in press.

Nigam, R., Kosovichev, A.G.: 1998, Measuring the sun's eigenfrequencies from velocity and intensity helioseismic spectra: Asymmetrical line profile-fitting formula. Astrophys. J. 505, L51. doi:10.1086/311594.

Provost, J., Berthomieu, G., Morel, P.: 2000, Low-frequency $p$ - and g-mode solar oscillations. Astron. Astrophys. 353, $775-785$.

Roca Cortés, T., Lazrek, M., Bertello, L., Thiery, S., Baudin, F., Boumier, P., Gavryusev, V., Garcia, R.A., Regulo, C., Ulrich, R.K., Grec, G., the GOLF Team: 1998, The solar acoustic spectrum as seen by GOLF. II. Noise statistics background and methods of analysis. In: Korzennik, S. (ed.) Structure and Dynamics of the Interior of the Sun and Sun-like Stars SP-418, ESA, Noordwijk, 323-334.

Scherrer, P.H., Bogart, R.S., Bush, R.I., Hoeksema, J.T., Kosovichev, A.G., Schou, J., Rosenberg, W., Springer, L., Tarbell, T.D., Title, A., Wolfson, C.J., Zayer, I., MDI Engineering Team: 1995, The solar oscillations investigation - Michelson Doppler Imager. Solar Phys. 162, 129-188. doi:10.1007/BF00733429.

Schou, J., Antia, H.M., Basu, S., Bogart, R.S., Bush, R.I., Chitre, S.M., Christensen-Dalsgaard, J., di Mauro, M.P., Dziembowski, W.A., Eff-Darwich, A., Gough, D.O., Haber, D.A., Hoeksema, J.T., Howe, R., Korzennik, S.G., Kosovichev, A.G., Larsen, R.M., Pijpers, F.P., Scherrer, P.H., Sekii, T., Tarbell, T.D., Title, A.M., Thompson, M.J., Toomre, J.: 1998, Helioseismic studies of differential rotation in the solar envelope by the solar oscillations investigation using the Michelson Doppler Imager. Astrophys. J. 505, 390-417. doi:10.1086/306146.

Thompson, M.J., Toomre, J., Anderson, E., Antia, H.M., Berthomieu, G., Burtonclay, D., Chitre, S.M., Christensen-Dalsgaard, J., Corbard, T., Derosa, M., Genovese, C.R., Gough, D.O., Haber, D.A., Harvey, J.W., Hill, F., Howe, R., Korzennik, S.G., Kosovichev, A.G., Leibacher, J.W., Pijpers, F.P., Provost, J., Rhodes, E.J., Schou, J., Sekii, T., Stark, P.B., Wilson, P.: 1996, Differential rotation and dynamics of the solar interior. Science 272, 1300-1305.

Toutain, T., Appourchaux, T.: 1994, Maximum likelihood estimators: An application to the estimation of the precision of helioseismic measurements. Astron. Astrophys. 289, 649-658. 